\title{
Modelo de Avaliação para Formação em Saúde baseada em Jogos e Ambientes Virtuais
}

\section{Assessment Model for Education in Health, based on Games and Virtual Environments.}

\author{
Modelo de Evaluación para la Formación Profesional \\ en Salud basada en Juegos y Ambientes Virtuales.
}

\author{
Thaíse K. L. Costa ${ }^{1}$ \\ Liliane S. Machado ${ }^{2}$ \\ Ana Maria G. Valença ${ }^{3}$ \\ Ronei M. Moraes ${ }^{4}$
}

\begin{abstract}
RESUMO: Este trabalho apresenta um estudo sobre processos de decisão em portais de jogos ou ambientes, e mostra um novo modelo de decisão que analisa o desempenho de estudantes, considerando os domínios cognitivo, afetivo e psicomotor. A partir de uma revisão, o presente trabalho revela que portais de jogos ou ambientes virtuais para a área de saúde podem incorporar processos avaliativos, baseados em modelos de decisão de máquina, que permitem avaliar conjuntamente atividades de apoio à formação de recursos humanos. Compreendendo que o planejamento dessas atividades deve ser balizado por objetivos educacionais, o presente trabalho apresenta um modelo de decisão de máquina baseado na Taxonomia dos Objetivos Educacionais. Este modelo de avaliação pode ser considerado como uma alternativa para avaliação de desempenho baseada em medidas objetivas de análise de desempenho nos portais de jogos e ambientes virtuais. Palavras chave: Avaliação Educacional; Técnicas de Apoio para a Decisão; Tecnologia Educacional.
\end{abstract}

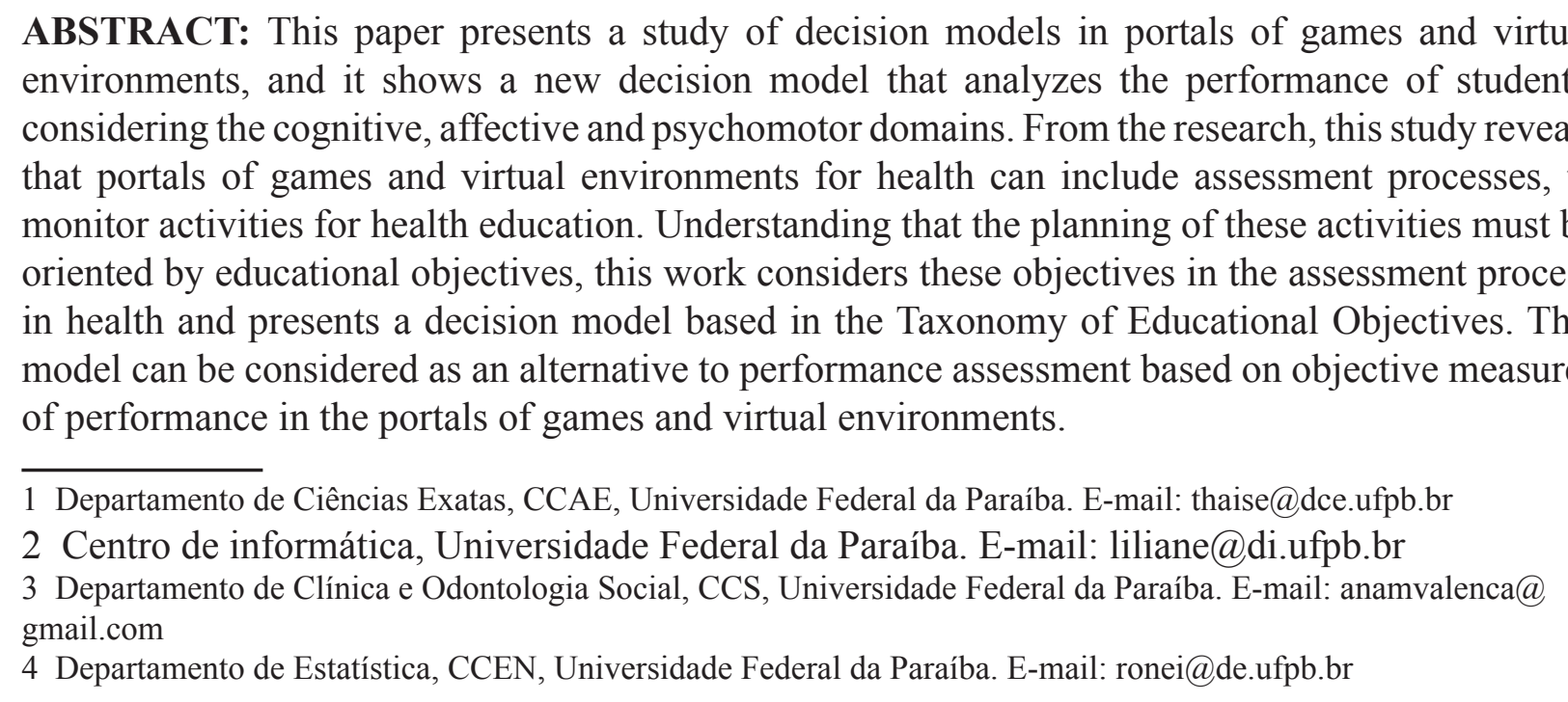


Keywords: Educational Measurement; Decision Support Techniques; Educational Technology.

RESUMEN: Este trabajo se presenta un estudio de los modelos de decisión en los portales de juegos y entornos virtuales, y se nota un nuevo modelo de decisión que analiza el rendimiento de los estudiantes, teniendo en cuenta los dominios cognitivos, afectivos y psicomotores. A partir de la investigación, este estudio revela que los portales de juegos y entornos virtuales para la salud pueden incluir los procesos de evaluación, para supervisar las actividades de educación para la salud. Entendiendo que la planificación de estas actividades debe estar orientada por objetivos educativos, este trabajo considera que estos objetivos en el proceso de evaluación en salud y presenta un modelo de decisión basado en la taxonomía de objetivos educativos. Este modelo puede ser considerado como una alternativa a la evaluación del desempeño basado en mediciones objetivas de desempeño en los portales de juegos y entornos virtuales.

Palabras clave: Evaluación Educacional; Técnicas de Apoyo para la Decisión; Tecnología Educacional.

\section{INTRODUÇÃO}

A formação em saúde envolve estudos, ações interdisciplinares e práticas em diferentes contextos. $\mathrm{O}$ avanço das tecnologias e dos meios de comunicação e interação com o mundo sugere mudanças no universo acadêmico, bem como na forma de orientar o processo de aprendizado, de promover a capacitação de recursos humanos e de incentivar a atuação prática. Nessa linha, os jogos e ambientes virtuais (por vezes chamados de simulações) permitem incluir no processo de formação, meios complementares aos métodos tradicionalmente utilizados de ensinar, aprender e praticar habilidades ${ }^{1}$.

Os jogos e ambientes no escopo do ensino e aprendizagem trazem vantagens para o exercício da prática de estudantes e profissionais da saúde, motivando sua aplicação na área. Eles, por exemplo, podem propiciar a experimentação de diferentes situações da vida profissional, com variação do nível de risco e tipos de materiais disponíveis. Além disso, eles podem fazer uso de processos de decisão que automatizam diferentes tarefas como a avaliação das decisões do usuário, o gerenciamento do grau de dificuldade do jogo ou simulação, o controle de oponentes (personagens virtuais), dentre outras ${ }^{2}$. De modo geral, pesquisas da área ratificam a importância desses recursos (jogos / ambientes) como meios de complementar o meio de construção de conhecimento, como em Nunes et al. ${ }^{3}$ e Taekman et al. ${ }^{1}$.

Os jogos e ambientes virtuais podem ser reunidos por meio de portais e plataformas, sendo estes considerados instrumentos que podem ajudar a centralizar a busca e acesso aos jogos e aos ambientes em um determinado contexto. Este fato desperta algumas indagações. Que serviços os portais ou plataformas de jogos têm a oferecer ao seu público? Como os processos de decisão são utilizados nesses portais? Como um modelo de decisão pode auxiliar no monitoramento de desempenho de usuários em portais de jogos e ambientes? Assim, o artigo tem por objetivo mostrar formas de utilização de processos de decisão de máquina na análise de informações, serviços oferecidos, bem como apresentar um novo modelo de avaliação, baseado em processo de decisão, para auxiliar o acompanhamento do usuário em portais de jogos e ambientes.

Tempus, actas de saúde colet, Brasília, 10(2), 253-273, jun, 2016.

ISSN 1982-8829 


\section{PERCURSO METODOLÓGICO}

A fim de apresentar um novo modelo avaliação para análise de desempenho de estudantes em portais de jogos e ambientes virtuais, este trabalho inicialmente mostra uma revisão acerca dos serviços oferecidos pelos portais. Nesse sentido, a revisão busca explorar e identificar os serviços e formas de utilização do processo de decisão nos portais e plataformas. Observou-se uma carência na disponibilização de trabalhos científicos que abordam o uso de plataformas e portais como instrumentos de integração de recursos ao currículo, sendo encontrados materiais disponíveis na web, não necessariamente em bases de publicação, razão pela qual a revisão desses portais não foi sistemática.

Considerando que o planejamento e a análise de atividades com fins educacionais devem ser balizados, respectivamente, pela definição e verificação dos objetivos educacionais da experiência, o atual trabalho considera importante a análise direta dos objetivos educacionais no processo de decisão que compõe uma avaliação. Assim, após a revisão, o artigo mostra e discute o uso da Taxonomia dos Objetivos Educacionais como um meio de padronização da informação em processo de decisão.

Por fim, com base na revisão acerca da utilização de processos de decisão em portais, bem como na importância da verificação do alcance dos objetivos educacionais em processos de decisão, o trabalho apresenta um novo modelo de avaliação apoiado na Taxonomia dos Objetivos Educacionais para analisar o desempenho de estudantes dentro de portais e plataformas de jogos e ambientes para formação em saúde.

Processo de decisão em portais de jogos e ambientes

Os processos de tomada de decisão estão presentes em atividades que impõem alternativas diversas com necessidade de análise para posterior posicionamento. Observa-se que cada vez mais aplicativos computacionais são desenvolvidos com possibilidade de auxiliar em processos de decisão por meio da Inteligência Artificial (IA) nos chamados modelos de decisão de máquina. A IA é uma área da Ciência da Computação que aponta variadas técnicas e recursos que permitem o desenvolvimento de programas aptos a tomar uma decisão de forma semelhante ao humano ${ }^{4}$.

No contexto dos jogos e Ambientes Virtuais (AVs), a IA pode atuar de diferentes maneiras, tais como na avaliação das decisões do usuário, no gerenciamento do grau de dificuldade do jogo ou simulação, e no controle de oponentes (personagens virtuais), dentre outras. $\mathrm{Na}$ área da saúde, destaca-se a utilização da IA para identificar o nível de conhecimento do usuário (avaliação do jogador) e, por meio deste nível de conhecimento, tomar decisões para avanços na aplicação (comportamento do jogo ou simulação) $)^{2}$.

Alguns portais de jogos e AVs também utilizam processos de decisão para auxiliar atividades. 
Esses instrumentos podem fornecer meios de acompanhamento dos usuários para identificar níveis de desempenho com auxílio da IA. Assim como nos jogos e AVs, a IA nos portais auxilia o processo de avaliação de usuários com possibilidade de tomada de decisão para definir mudança em seu ambiente.

Nessa linha de portais, o Portal of Instructional Games, Online (Pingo) disponibiliza jogos educacionais com conteúdo customizável e possui um repositório com temas que abrangem várias disciplinas do ensino básico, permitindo que professores de escolas brasileiras personalizem seus conteúdos. O acesso ao ambiente é livre e específico para cada perfil de usuário: desenvolvedor de jogos, professor e aluno. Utilizando o portal, os docentes podem selecionar jogos por meio do repositório, adaptar o conteúdo ao seu contexto de trabalho e disponibilizá-los, individualmente, para suas turmas. Os alunos, por sua vez, têm acesso aos jogos customizados, sendo monitorados pelo ambiente que realiza o registro de dados em cada jogo (tempo gasto, pontuação, erros e acertos), que podem ser visualizados pelo docente ou pelo próprio discente ${ }^{5}$ O portal realiza a análise de desempenho dos alunos de cada professor e auxilia o docente no gerenciamento da sua turma.

Também com foco no público infantil, o portal Attractive Virtual Educacional Portal (AVEP) disponibiliza jogos 2D (matemática, línguas, lógica, dentre outras), mas possui acesso restrito para testes feito com escolas da República Eslovaca e Checa. Ele oferece a possibilidade de inserção de novos jogos e apresenta um serviço de acompanhamento do aluno com base em dados obtidos pelo instrumento. A partir dos dados registrados, o portal realiza uma análise automática, por meio de IA, que permite ao professor acompanhar o desempenho do aluno e complementar tarefas ${ }^{6}$.

Na linha de plataformas para dispositivos móveis, pode-se citar o mobile Game Based Learning ${ }^{7}$ (mGBL), uma plataforma que permite centralizar a exposição e acesso a materiais educativos. Ela dispõe de três módulos, sendo eles: de autoria, de distribuição e de monitoramento. O módulo de autoria permite aos professores a construção de jogos a partir de modelos. O módulo de distribuição assegura a disponibilização da plataforma em diferentes redes. Já o módulo de monitoramento utiliza técnicas de IA para controlar o jogo e avaliar o sucesso da aprendizagem?

Bamidis et al. ${ }^{8}$ relatam o desenvolvimento de um portal para auxílio a tratamentos, que reúne diferentes exercícios cognitivos para evitar o declínio da memória, juntamente com atividades físicas, incluindo jogos, para promover assistência avançada a idosos. Por meio do aplicativo, os terapeutas podem esquematizar programas de treinamento personalizados, visualizar o andamento das sessões, bem como, adicionar novos jogos e exercícios no sistema. O portal possui três tipos de usuários: idosos, familiares e terapeutas. Os idosos têm acesso ao seu programa de tratamento e pode realizar os exercícios. Os familiares podem acessar o sistema para acompanhar o progresso do tratamento do seu idoso. Já os terapeutas podem construir o roteiro de tratamento, ajustar a 
intensidade e duração do treinamento, bem como acompanhar o desempenho dos seus pacientes a partir dos resultados disponibilizados pela IA.

Todos esses portais e plataformas buscam, de alguma forma, reunir jogos e simulações como estratégias educacionais para facilitar a exploração e organização do uso desses recursos com grupos de usuários. Por meio delas, a busca e a seleção de recursos (jogos e AVs) podem ser facilitadas, auxiliando o planejamento de atividades. A partir dos trabalhos apresentados identificam-se alguns tipos de serviços, como:

- Armazenamento: serviço responsável pelo registro de informações e pesquisa dos jogos e simulações no instrumento;

- Ajuste ou Acréscimo: serviço responsável por permitir a inserção, adaptação, ou criação de novos jogos e simulações por meio do portal;

- Projeção de atividades: serviço que possibilita aos mediadores a criação de atividades, que inclui a seleção de jogos ou simulações para os aprendizes/pacientes;

- Monitoramento: serviço responsável pelo registro de desempenho dos aprendizes/pacientes, com possível geração de relatório.

Como o foco do atual trabalho é o processo de decisão, destacamos aqui o serviço de monitoramento, que envolve o uso de processos de decisão de máquina, ou seja, IA, para auxiliar a verificação do desempenho do usuário. Esse serviço pode fornecer as informações de desempenho de duas formas: (i) conferir informações acerca do desempenho do usuário por jogo ou simulação, ou (ii) conferir informações de desempenho analisando aspectos de vários jogos ou simulações para uma avaliação conjugada (geral), podendo ser útil no gerenciamento de atividades.

É válido ressaltar que nem todos os portais de jogos ou ambientes virtuais dispõem de serviço de monitoramento ou serviços que envolvem processos de decisão de máquina ${ }^{9,10}$. Nesse contexto, tem-se o portal Games and Simulation for Healthcare Library and Database que provê uma lista de jogos e ambientes para atender as necessidades de educadores da saúde, pesquisadores, profissionais e pacientes que desejam conhecer jogos e simulações para serem usados como estratégia para ensino e assistência ao paciente. O portal disponibiliza um inventário com jogos e simulações que permite apenas a visualização de detalhes de cada aplicativo e o endereço para $\operatorname{acesso~}^{10}$.

O presente trabalho destaca a importância da tomada de decisão de máquina no monitoramento de usuários de portais para promover a análise de desempenho em atividades do processo de formação em saúde. A Quadro 1 mostra as formas de monitoramento observadas nos portais apresentados nesse trabalho. 
Quadro 1 - Presença do serviço de monitoramento no(a)s plataformas/portais avaliados.

\begin{tabular}{|c|c|c|}
\hline \multirow{2}{*}{ Plataforma/Portal } & \multicolumn{2}{|c|}{ Monitoramento do Usuário } \\
\cline { 2 - 3 } & $\begin{array}{c}\text { Verificação do desempenho por jogo } \\
\text { ou simulação }\end{array}$ & $\begin{array}{c}\text { Verificação do desempenho conjunto } \\
\text { nos jogos ou simulações }\end{array}$ \\
\hline Pingo & Sim & Não \\
\hline AVEP & Sim & - \\
\hline mGBL & Sim & Não \\
\hline Plataforma de tratamento & Sim & - \\
\hline
\end{tabular}

Fonte: elaborado pelo autor.

No Quadro 1, o valor nulo (“-“)representa a falta de informações explícitas sobre a característica analisada. Assim, nota-se que os portais apresentados fornecem o serviço de monitoramento de usuário por jogo ou simulação; podendo ser esse monitoramento oferecido pelos próprios recursos ou provido pelos portais. Observa-se que a análise de desempenho do usuário considerando aspectos conjunto nos jogos ou simulações para prover avaliação mais geral ainda não é contemplada nesses portais, o que sugere que a possibilidade de inclusão e adaptação desse serviço é um espaço de pesquisa em potencial.

Nas pesquisas, não foram encontrados portais de jogos e AVs para formação profissional em saúde que utilizam serviço de monitoramento. Porém, a inclusão de processos de decisão de máquina nesses portais de saúde permitiria acompanhar e avaliar o desempenho dos usuários (estudantes), auxiliando mediadores no processo de formação profissional baseada no uso de jogos e ambientes virtuais. A inclusão desse e outros serviços nos portais de jogos e AVs contribui para o uso desses instrumentos no apoio à formação e afasta a concepção de que os portais são utilizados apenas para armazenamento de recursos.

Taxonomia dos objetivos educacionais para padronização da informação em processo de decisão

A utilização de processos de decisão a fim de prover serviços de monitoramento e avaliação em portais, requer que as informações utilizadas para análise nos diferentes jogos e ambientes possuam um mesmo padrão. No caso dos portais de jogos e AVs para a formação em saúde, a análise de desempenho dos estudantes pode ser feita a partir da averiguação de informações relativas ao alcance dos objetivos educacionais ${ }^{11}$.

Conforme afirmam Anderson et al. ${ }^{12}$, o resultado do ensino se concretiza a partir dos objetivos, portanto, estes devem ser considerados tanto no planejamento quanto na avaliação. Por natureza, os objetivos são de curto prazo e devem ser atingíveis na conclusão de uma sessão de ensino 
ou, geralmente, dentro de um período que inclui uma série de sessões de ensino (sequência de atividades). Os objetivos podem ser vistos como declarações que informam ao estudante o que é esperado sob uma perspectiva cognitiva, afetiva e psicomotora, sendo anterior a meta, que é o resultado maior ${ }^{11}$.

Para portais de jogos e AVs, a análise de desempenho do usuário pode considerar objetivos distribuídos entre os diferentes aplicativos que, nesse caso, necessitam ter uma linguagem de declaração unificada. Assim, é importante a utilização de subsídios que permitam a classificação desses objetivos dentro de um portal composto por diferentes jogos e simulações como forma de padronizar a linguagem de comunicação. Tal fato possibilita a realização de análises e aplicação de processos de decisão a partir da verificação dos objetivos contemplados em diferentes aplicativos de um mesmo portal.

De acordo com Ferraz et al. ${ }^{13}$, existem subsídios que apoiam o planejamento didático-pedagógico, a estruturação, a organização, a definição dos objetivos e a escolha de meios de avaliação. A Taxonomia dos Objetivos Educacionais, também conhecida como Taxonomia de Bloom, é um desses subsídios que possuem como finalidade o auxílio na declaração e identificação dos objetivos educacionais. Ainda de acordo com Ferraz et al. ${ }^{13}$, muitos são os proveitos na utilização da Taxonomia de Bloom no âmbito educacional. Dentre elas destaca-se o fornecimento da base para desenvolver meios de avaliação e emprego de estratégias diferenciadas para favorecer, avaliar e incentivar o desempenho dos alunos em níveis de aquisição de conhecimento. Com isso, tornase possível categorizar os objetivos educacionais de forma clara e significativa, apresentando um objetivo educacional de forma similar para diferentes pessoas e em diferentes contextos, permitindo inferências sobre os tipos de comportamentos esperados dos estudantes.

A Taxonomia de Bloom (ou taxonomia dos objetivos educacionais) proporciona um sistema conveniente para a descrição e ordenação de itens de teste, técnicas de exame e instrumentos de avaliação. Além disso, permite comparar e estudar programas educacionais, observando a similaridade dos objetivos e experiências de aprendizagem. Nessa taxonomia os tipos de aprendizado são diferenciados em três domínios: cognitivo, afetivo e psicomotor. Para Bloom et al. ${ }^{14}$ a soma desses três domínios resulta no desenvolvimento integral da pessoa.

O domínio cognitivo envolve o aprender e inclui a obtenção de um novo conhecimento, habilidade intelectual, compreensão, reflexão e atitude sobre um problema ou fato. Ele foi reformulado por um grupo de especialistas supervisionado por Krathwohl, um dos autores da primeira formulação. Essa reformulação manteve a ideia base do domínio cognitivo e o dividiu em duas dimensões: dimensão do processo cognitivo e dimensão do conhecimento. No entanto, apenas a dimensão do processo cognitivo se tornou mais conhecida e aplicada no contexto acadêmico. Essa dimensão está relacionada ao processo, ou seja, "como" algo será alcançado, representado a organização 
da complexidade cognitiva de forma crescente. Ela foi dividida em seis categorias hierárquicas: lembrar, entender, aplicar, analisar, avaliar e criar.

O domínio afetivo está relacionado aos sentimentos, posturas, reações de ordem afetiva e de empatia. Ele pode ser demonstrado por comportamentos e atitudes de consciência/sensibilização, interesse, atenção, inquietação/preocupação, responsabilidade, capacidade para ouvir e responder em interações com os outros, bem como capacidade de demonstrar essas características comportamentais ou valores apropriados a cada tipo de teste e campo de estudo ${ }^{15}$. Ele é dividido em cinco categorias hierárquicas: receptividade, resposta, valorização, organização e caracterização por um valor ou grupo de valores.

Já o domínio psicomotor envolve habilidades que combinam ações musculares e cognição. Logo, esse domínio relaciona habilidades físicas e processos cognitivos, tratando de habilidades ligadas ao manipular ferramentas ou objetos. Bloom e sua equipe não desenvolveram uma taxonomia para a área psicomotora. Como resultado, existem diferentes contribuições advindas de trabalhos nesse terceiro domínio, por exemplo, o domínio psicomotor proposto por Dave ${ }^{16}$, bastante difundido, simples e apropriado para a maioria das formações de adultos na área de saúde ${ }^{11}$. O Domínio Psicomotor de Dave sugere que a precisão deve ser salientada antes que a velocidade na qual uma habilidade é adquirida ${ }^{17}$. Ele é dividido em cinco categorias, sendo elas: imitação, manipulação, precisão, articulação e naturalização.

Segundo Conklin ${ }^{18}$, a taxonomia dos objetivos educacionais, com sua classificação hierárquica, avanços na reformulação e projetos de continuação, é de grande contribuição para os educadores. O uso consciente desse sistema oferece meios de estimular raciocínios e abstrações de alto nível, a partir de objetivos.

$\mathrm{Na}$ saúde, os objetivos da formação expressam onde o aluno, submetido a situações de aprendizagem, poderá chegar. Por exemplo, ao ser capaz de classificar categorias de pacientes por complexidade de uma fratura no fêmur o estudante ou profissional demonstra ter compreensão acerca do conteúdo envolvido. Portanto, de acordo com a taxonomia, ele alcança o "entender" do domínio cognitivo. Ao demonstrar iniciativa para desenvolver um plano de atuação de equipe para cuidado com pacientes, analisando o aspecto afetivo desse objetivo, o profissional demonstra “organização". Já ao ser capaz de combinar ações ao manipular ferramentas para atender nova necessidade na execução de um procedimento, pode-se dizer que o profissional demonstra "articulação" para o domínio psicomotor. A taxonomia, nesse sentido, sistematiza os objetivos verificando complexidade, abstração e internalização.

Uma das vantagens de utilização desse sistema de classificação consiste no fato de não possuir delimitação por nenhuma modalidade ou estratégia educacional, assim, a preocupação restringe-se Tempus, actas de saúde colet, Brasília, 10(2), 253-273, jun, 2016.

ISSN 1982-8829 
à efetividade do processo. Dessa forma, a taxonomia demonstra seu relacionamento com o "como" implementar objetivos, em vez de restringir formas ou ambientes nos quais a aprendizagem pode acontecer ${ }^{13}$.

Apesar de a taxonomia ter sido proposta há algumas décadas, ela permanece atual no contexto educacional por ser base para o desenvolvimento de instrumentos de avaliação e considerar diferentes níveis de aquisição de conhecimento em domínios distintos. Essa categorização ajuda programas de capacitação e treinamento a deixarem claros seus objetivos, bem como o foco da avaliação. Da mesma forma, os jogos e ambientes virtuais, possuem um conjunto de objetivos que podem ser classificados com base na taxonomia, ajudando principalmente em dois aspectos: seleção e avaliação. Com isso, é possível utilizar medidas e técnicas de IA para verificar o desempenho do estudante ao longo de uma sequência de atividades.

Um modelo de avaliação de desempenho geral

Dentro do contexto de avaliação em portais de jogos, apresentamos um modelo de avaliação que se apoia na Taxonomia dos Objetivos Educacionais para analisar o desempenho dos estudantes durante a realização de uma sequência de atividades elaborada pelo mediador e composta por diferentes jogos ou ambientes virtuais. Cada mediador, ao criar a sequência de atividades poderá customizar o processo de avaliação, selecionando os objetivos de aprendizagem almejados para a sequência específica. Dessa forma, a partir de informações enviadas pelos jogos e ambientes virtuais, o módulo de avaliação realizará a análise e tomada de decisão acerca do desempenho discente.

A avaliação considera que uma sequência de atividades é composta por níveis hierárquicos, formados por um conjunto de jogos e/ou simulações (Figura 1). Por serem hierárquicos, a passagem para um nível posterior só será realizada ao cumprir as exigências mínimas do nível atual. Essas exigências são estipuladas pelos mediadores, sendo representadas por valores mínimos de desempenho em categorias dos domínios de aprendizagem, dependendo dos objetivos pretendidos. Ao concluir a sequência, os discentes demonstrarão terem cumprido as exigências mínimas e alcançado seu propósito. 
Figura 1 - Composição geral de uma sequência de atividades.

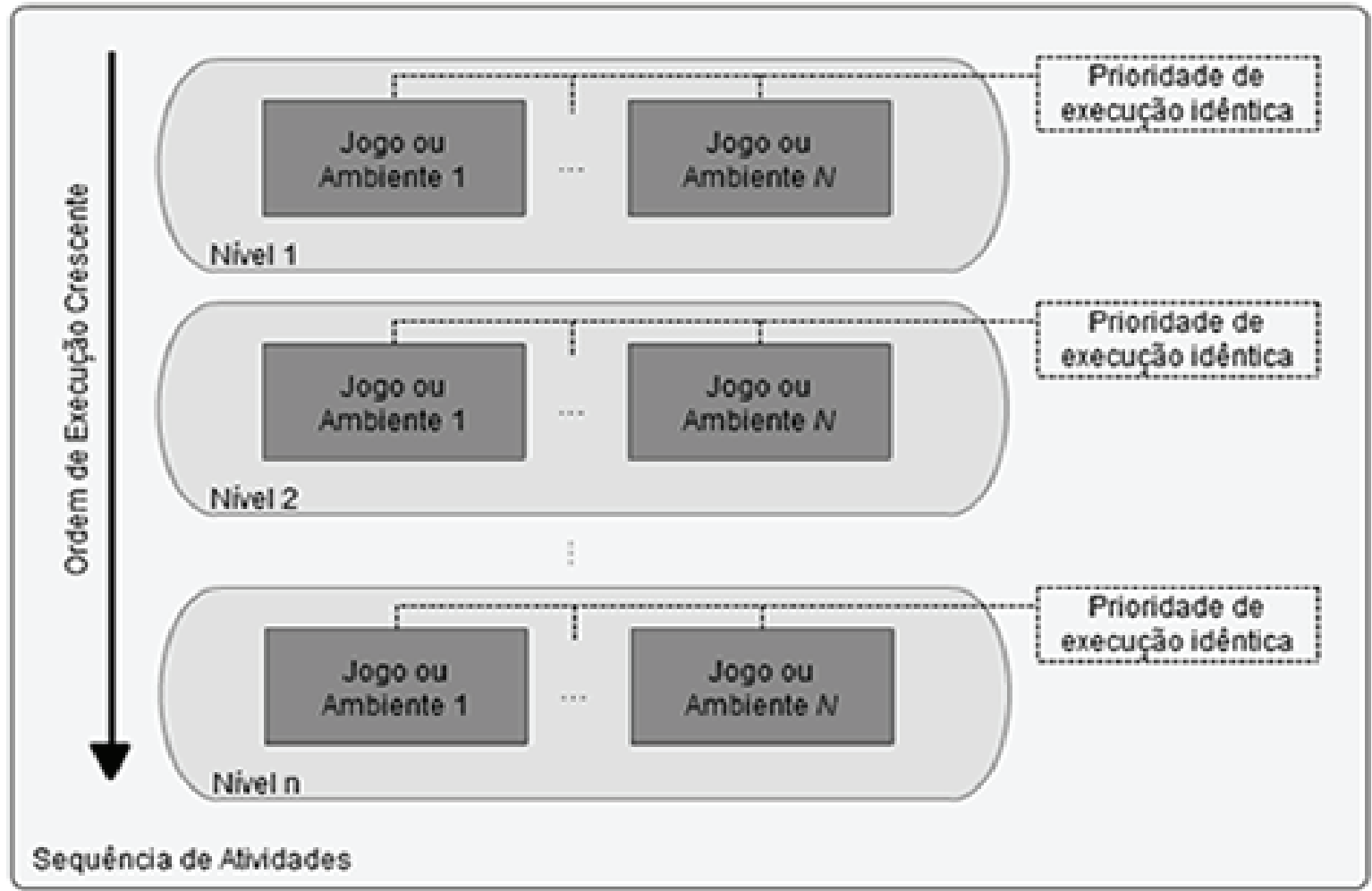

Assim, para explicar a proposta do modelo de avaliação para formação baseada em jogos e ambientes virtuais, inicialmente será apresentado o objeto alvo da análise nas sequências de atividades. Em seguida, serão mostradas as necessidades e restrições do módulo de avaliação. Por fim, será exposto o método pelo qual se realizará a verificação e tomada de decisão acerca do desempenho.

Objeto de análise do módulo de avaliação

O módulo de avaliação verifica o desempenho dos educandos em cada nível da sequência de atividades. O desempenho é medido com base no cumprimento dos objetivos educacionais estabelecidos para cada nível. A definição dos objetivos é feita pelo mediador em dois passos:

1. Seleção das categorias dos domínios de aprendizagem que se deseja avaliar em cada jogo ou ambiente. Essas categorias representam os objetivos educacionais que o mediador deseja trabalhar com seus mediados;

2. Determinação do grau de suficiência mínimo no cumprimento de cada objetivo educacional estabelecido. Esse grau de suficiência é representado por um valor mínimo de sucesso (descrito em porcentagem) que um discente deve obter em cada categoria que será avaliada (relevância do objetivo no planejamento).

Assim, quando um usuário finaliza um jogo/ambiente contido em um dado nível da sequência de atividades, a informação de sucesso ou fracasso nesse aplicativo é utilizada pelo módulo de avaliação para o processo de análise de desempenho do estudante no contexto daquele nível. Um 
exemplo do processo de definição dos objetivos pelo mediador é exibido por meio das Figuras 2 e 3.

A Figura 2 mostra um suposto nível de uma sequência composta por quatro atividades (jogos ou ambientes). Observa-se que a atividade 1 possui seis objetivos educacionais que foram classificados em categorias presentes nos domínios cognitivo e psicomotor. A atividade 2 possui oito objetivos educacionais que foram classificados em categorias presentes nos três domínios. Já a atividade 3 possui nove e a atividade 4 possui oito objetivos educacionais que também foram classificados em categorias presentes nos três domínios.

O processo de definição dos objetivos para esse nível da sequência de atividades inicia com a escolha das categorias dos domínios de aprendizagem que serão avaliadas em cada jogo ou ambiente. Na Figura 3, percebe-se que apenas parte dos objetivos disponíveis foi selecionada para participar da avaliação nesse primeiro nível da sequência. Tal fato representa a opção do mediador em focar a avaliação desse nível em determinados aspectos que considera relevante para o contexto.

Após o primeiro passo, o mediador definirá as condições mínimas que permitem dizer que o educando passou de nível. Essas condições são representadas por porcentagens mínimas de sucesso exigidas para cumprimento de cada categoria.

Ainda na Figura 3 são apresentados os valores especificados para conclusão do nível 1. Nessa Figura, observa-se a definição do valor de 50\% para a categoria "aplicar" do domínio cognitivo. Assim, o mediador estará afirmando que espera que o aluno cumpra pelo menos metade das atividades que analisam essa categoria, a fim de ser considerado apto a passar de nível. Como no exemplo da Figura 3 a categoria "aplicar" foi selecionada apenas nas atividades 1 e 3, então é necessário que o aluno obtenha sucesso em uma das duas atividades para alcançar os $50 \%$ de rendimento e ser considerado apto no "aplicar" do domínio cognitivo.

Figura 2 - Exemplo de sequência com primeiro nível composto por quatro atividades: exibição dos objetivos de cada jogo.

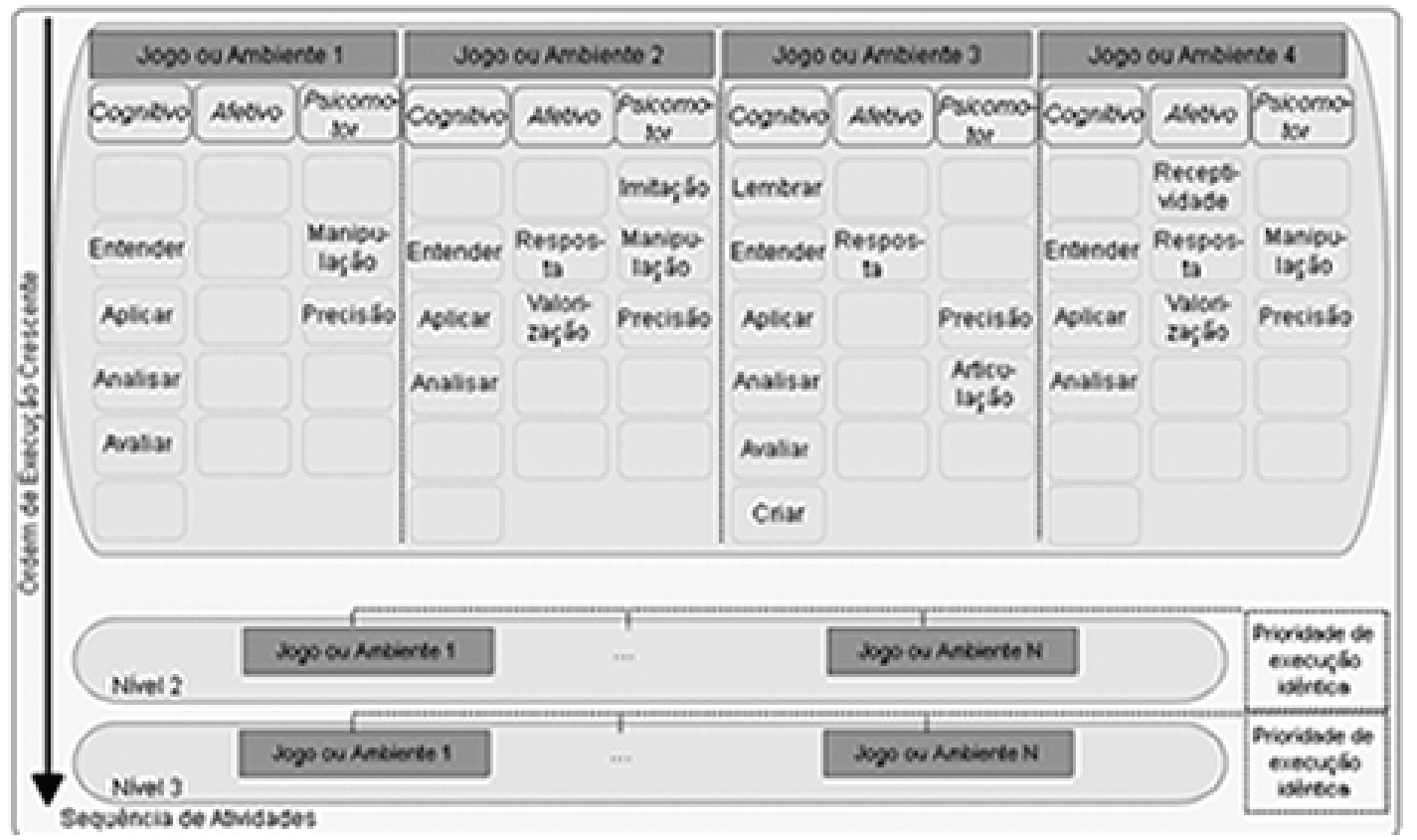


Figura 3 - Exemplo de sequência com primeiro nível composto por quatro atividades: definição do desempenho mínimo por categoria.

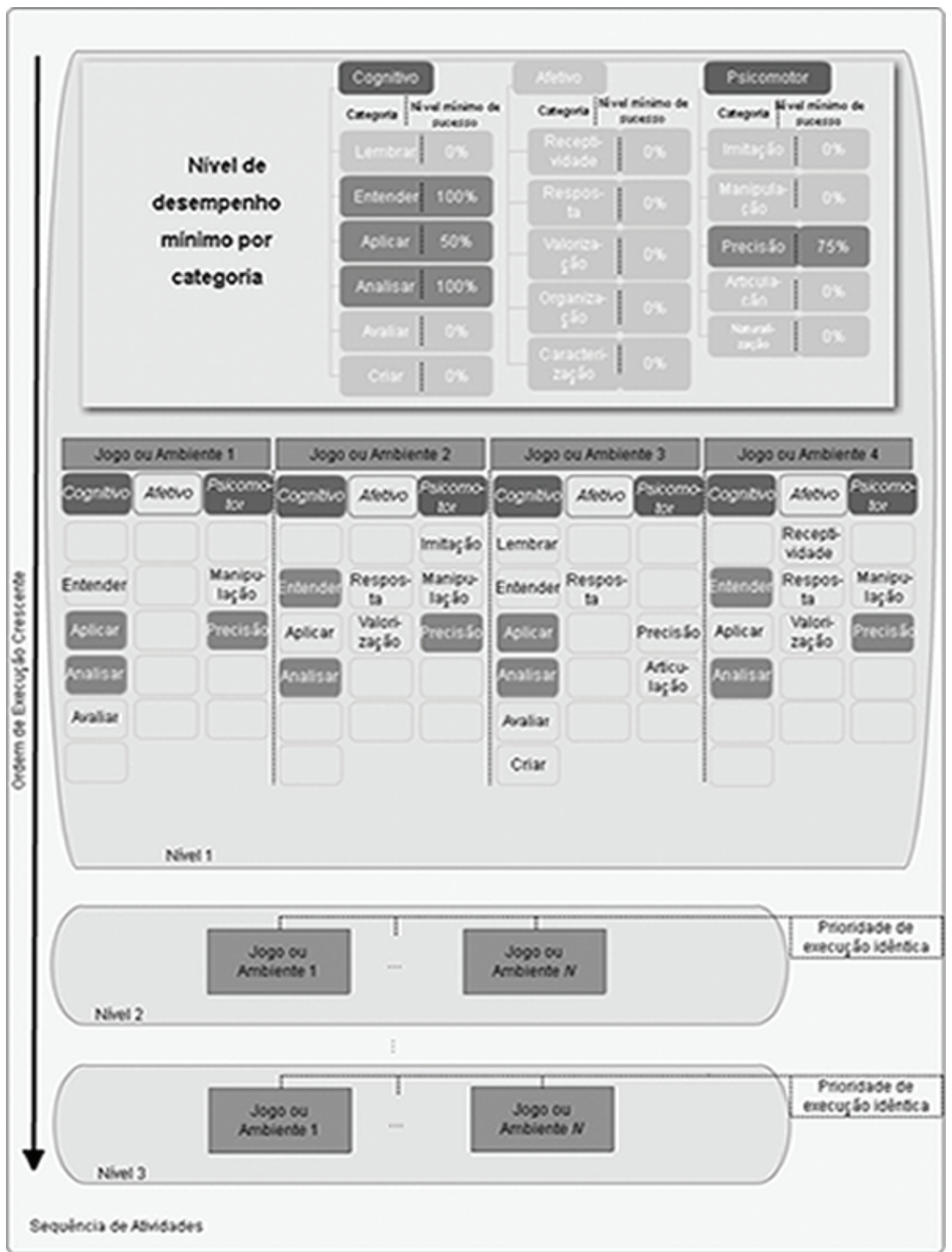

Restrições do módulo de avaliação

Os jogos e ambientes virtuais que compõem a sequência de atividades devem considerar dois pontos importantes: 
i. Apresentar os objetivos educacionais de forma clara e concisa.

ii. Retornar a informação de "sucesso" ou "fracasso" do usuário após a finalização do aplicativo. Essa informação deverá ser fornecida ao módulo de avaliação, com base em uma avaliação particular (interna) do aplicativo que analisa o cumprimento dos objetivos educacionais determinados.

Por considerar estes pontos, os objetivos declarados pelos jogos e AVS necessitam ser classificados de acordo com a Taxonomia dos Objetivos Educacionais ${ }^{14-15,19}$. Na seção anterior foi visto que os domínios de aprendizagem são divididos em categorias, permitindo a classificação dos objetivos. A Figura 4 apresenta um resumo dos domínios e categorias presentes na taxonomia e utilizados pelo módulo de avaliação do portal.

A partir desse padrão, diferentes jogos e ambientes podem classificar seus variados objetivos em categorias específicas dentro dos domínios de aprendizagem (Cognitivo, Afetivo e Psicomotor). Com isso, é possível utilizar medidas e técnicas de inteligência artificial para verificar o desempenho do usuário ao longo da sequência de atividades.

Figura 4 - Resumo dos domínios de aprendizagem e suas respectivas categorias.
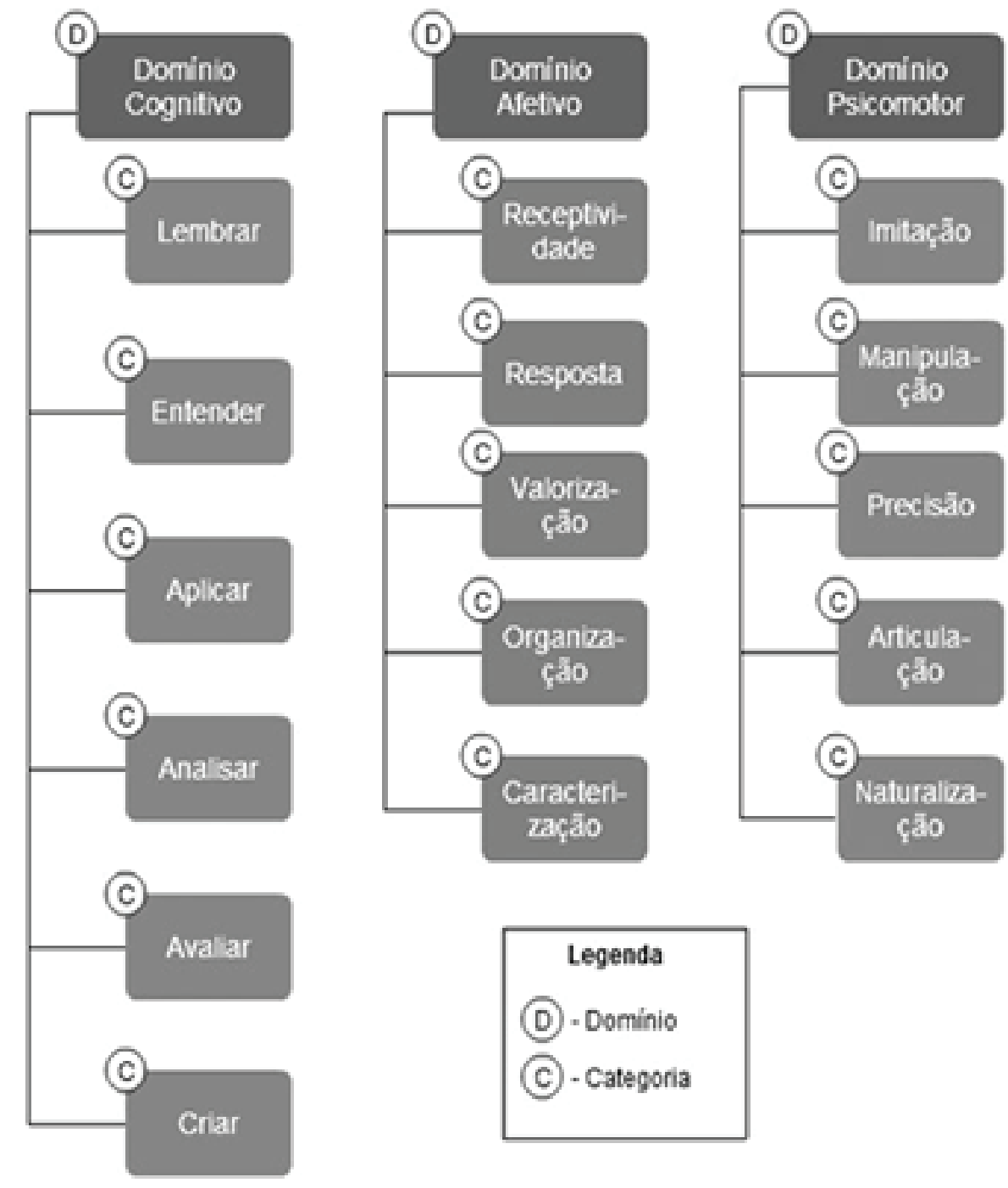
O Quadro 2 apresenta um exemplo fictício de jogo que poderia ser incorporado ao portal. Esse suposto jogo avalia o desempenho considerando três objetivos que o usuário deve alcançar para a obtenção de sucesso, sendo eles:

1. Executar o protocolo de tomada de decisão adequado;

2. Determinar corretamente se os pacientes estão fisicamente e mentalmente preparados;

3. Manter a ética durante o procedimento.

Quadro 2 - Exemplo de classificação dos objetivos educacionais de um suposto jogo.

\begin{tabular}{|l|l|}
\hline Jogo: & Decisões de implante dentário \\
\hline \multicolumn{2}{|c|}{$\begin{array}{l}\text { Descrição: jogo 3D no qual os alunos guiam seu avatar e interagem com os pacientes virtuais para praticar o } \\
\text { protocolo de tomada de decisão apropriado para determinar se os pacientes estão fisicamente e mentalmente }\end{array}$} \\
preparados para procedimentos de implantes dentários. \\
\hline & \multicolumn{2}{|c|}{ Objetivo do jogo } & \multicolumn{1}{c|}{ Classificação } \\
\end{tabular}

Fonte: elaborado pelos autores.

Cada um desses objetivos são supostamente analisados para o cálculo da avaliação interna do jogo/ambiente, por isso foram classificados na Taxonomia dos Objetivos Educacionais, conforme apresentado no Quadro 2. Ao retornar "sucesso", o jogo garante que os objetivos foram alcançados pelo usuário, já ao retornar "fracasso", afirma que o usuário necessita refazer a atividade. É importante ressaltar que a avaliação interna é uma obrigação do jogo/ambiente, sendo utilizada pelo portal como um fator para análise de desempenho em um contexto de maior abrangência.

Método de análise do desempenho

O processo de análise e tomada de decisão no módulo de avaliação é realizado por meio de um Sistema Baseado em Regras (SBR). O SBR consiste em um conjunto de declarações que formam uma "memória de trabalho" e um conjunto de regras que direcionam como agir de acordo com as declarações. A estratégia utilizada nesse sistema especialista é que o conhecimento de um perito está codificado em um conjunto de regras. Dessa forma, o sistema baseado em regras deve agir de forma semelhante ao perito ${ }^{20}$.

Geralmente, esses sistemas são simples modelos que podem ser adaptados para diferentes casos, em especial para casos nos quais todo o conhecimento na área pode ser descrito através de regras. De acordo com Millington et al. ${ }^{21}$, a criação de um SBR deve considerar um conjunto relevante de fatos, que formam a base de conhecimento; e representados por regras, que englobam 


\section{$267 / /$}

toda e qualquer ação possível. As regras são compostas por condições que devem ser analisadas para direcionar ações determinadas.

$\mathrm{Na}$ execução do comportamento representado pelas regras, todo o conjunto é percorrido e, para cada uma delas, a situação do ambiente é avaliada para verificar se deve disparar. Caso positivo, o comportamento será aquele descrito pela regra. Dessa forma, o módulo de avaliação realiza os cálculos de desempenho em um dado nível e, em seguida, verifica se as regras foram cumpridas. Caso afirmativo o jogador passa para o nível seguinte, caso negativo permanece no mesmo nível.

A avaliação é adaptável, ou seja, para cada nível da sequência de atividades, o mediador pode selecionar os atributos que serão considerados no cálculo do desempenho. Assim, conforme apresentado anteriormente, o mediador precisará (para cada nível):

1. selecionar quais categorias dos domínios serão consideradas em cada jogo;

2. definir valores mínimos (porcentagens mínimas) de sucesso nas categorias que foram selecionadas, sendo importante ressaltar que esses valores são únicos para cada categoria, ou seja, definidos uma vez em um nível e independente de jogo.

As regras serão aplicadas com base nesses atributos estabelecidos pelo mediador. Assim, para cada categoria selecionada na avaliação:

- realizar-se-á o cálculo de porcentagem de sucesso do jogador na categoria;

- $\quad$ verificará se regra foi cumprida.

O algoritmo é baseado em regras que devem ser aplicadas em cada nível da sequência de atividades, sendo expresso por meio do pseudocódigo apresentado na Figura 5.

Figura 5 - Pseudocódigo para análise e tomada de decisão acerca do desempenho do usuário.

1. Para o Nivel

1.1. Para cada Domínio

1.1.1. Se o Dominio foi selecionado para ser avaliado, então executa passo 1.1.2, senăo volta para passo 1.1 .

1.12. Para cada Categoria selecionada no Dominio

1.1.2.1. Verifica em quantos jogos ela foi selecionada para ser avaliada (valor 1) e em quantos desses jogos que ela foi selecionada 0 usuário obteve sucesso (valor 2).

1.1.2.2 Faz o calculo da porcentagem de sucesso, dividindo o valor 2 pelo valor 1 , multiplicando por 100 .

1.1.2.3 Verifica se o valor calculado é igual ou superior à porcentagem minima exigida para passar de nível. Caso afirmativo, então passa na categoria, senăo năo passa na categoria.

1.1.3. Se passou em todas as Categorias avaliadas ou verificou que nenhuma Categoria foi selecionada para ser avaliada, entăo passou no Domínio, senåo năo passou no Domínio.

12. Se passou em todos os Dominios avaliados ou verificou que nenhum Dominio foi selecionado para ser avaliado, então passou de nível, senăo năo passou de nível. 


\section{$268 / /$}

O SBR foi escolhido como técnica de análise devido à sua simplicidade e eficácia para a temática proposta. Ele permite que o método de avaliação apresentado conceda autonomia ao docente para adaptar a análise aos objetivos do seu planejamento, ao mesmo tempo em que automatiza o acompanhamento do aluno durante a realização da sequência de atividades.

Caso de uso do modelo de avaliação

A fim de exemplificar o uso do modelo de avaliação apresentado neste artigo, esta seção esquematiza uma sequência de atividade que apoia o aprendizado para administração de anestesia. Para isso, utilizaram-se cinco jogos organizados em uma sequência composta por três níveis, como ilustrado na Figura 6.

No primeiro nível encontram-se os jogos A e B. O jogo A possui como objetivo geral o apoio ao aprendizado da anatomia e de técnicas de administração de medicamentos injetáveis, focando nos níveis iniciais do domínio cognitivo. Já o jogo B possui como objetivo geral o apoio ao aprendizado de equipamentos e técnicas para a administração de medicamentos injetáveis, também focando nos níveis iniciais do domínio cognitivo. Esses jogos foram agrupados em um único nível a fim de facilitar a capacitação acerca da anatomia, equipamentos e técnicas para administração de medicamentos injetáveis.

No segundo nível encontram-se os jogos C e D. O jogo C possui como objetivo geral o apoio ao aprendizado acerca do procedimento de administração de medicamento injetável, focando não só na aplicação como também em aspectos psicomotores. O jogo D possui como objetivo geral o apoio ao aprendizado do procedimento de higienização das mãos, focando também a aplicação dos procedimentos, bem como aspectos psicomotores. No nível 2, os jogos reunidos procuram possibilitar a prática individual da aplicação de procedimentos, uma vez que conceitos mais básicos já foram exercitados no nível 1.

No terceiro nível encontra-se o jogo E, que possui como objetivo a administração de anestesia durante procedimento realizado com equipe cirúrgica. Esse jogo proporciona a aplicação e análise da administração de anestesia no contexto de trabalho em equipe.

Para cada nível foram (1) selecionadas as categorias dos domínios consideradas no módulo de avaliação e (2) definidos os valores mínimos (porcentagens mínimas) de sucesso nas categorias selecionadas (Figura 6). No caso de uso, para passar no primeiro nível o discente deve ser capaz de lembrar-se dos conteúdos abordados em todos os jogos daquele nível (Lembrar=100\%) e demonstrar que entendeu o conteúdo abordado em, ao menos, metade dos jogos (Entender=50\%).

Para passar do nível 2, o discente tem que ser capaz de aplicar e de ser preciso em todos os jogos que compõem o nível (Aplicar $=100 \%$ e Precisão=100\%). Por último, no nível 3, o aluno necessita mostrar que sabe aplicar o procedimento no contexto de trabalho em equipe (Aplicar=100\%). 
Figura 6- Caso de uso: sequência de atividades composta por três níveis apoiar o aprendizado da administração de anestesia.

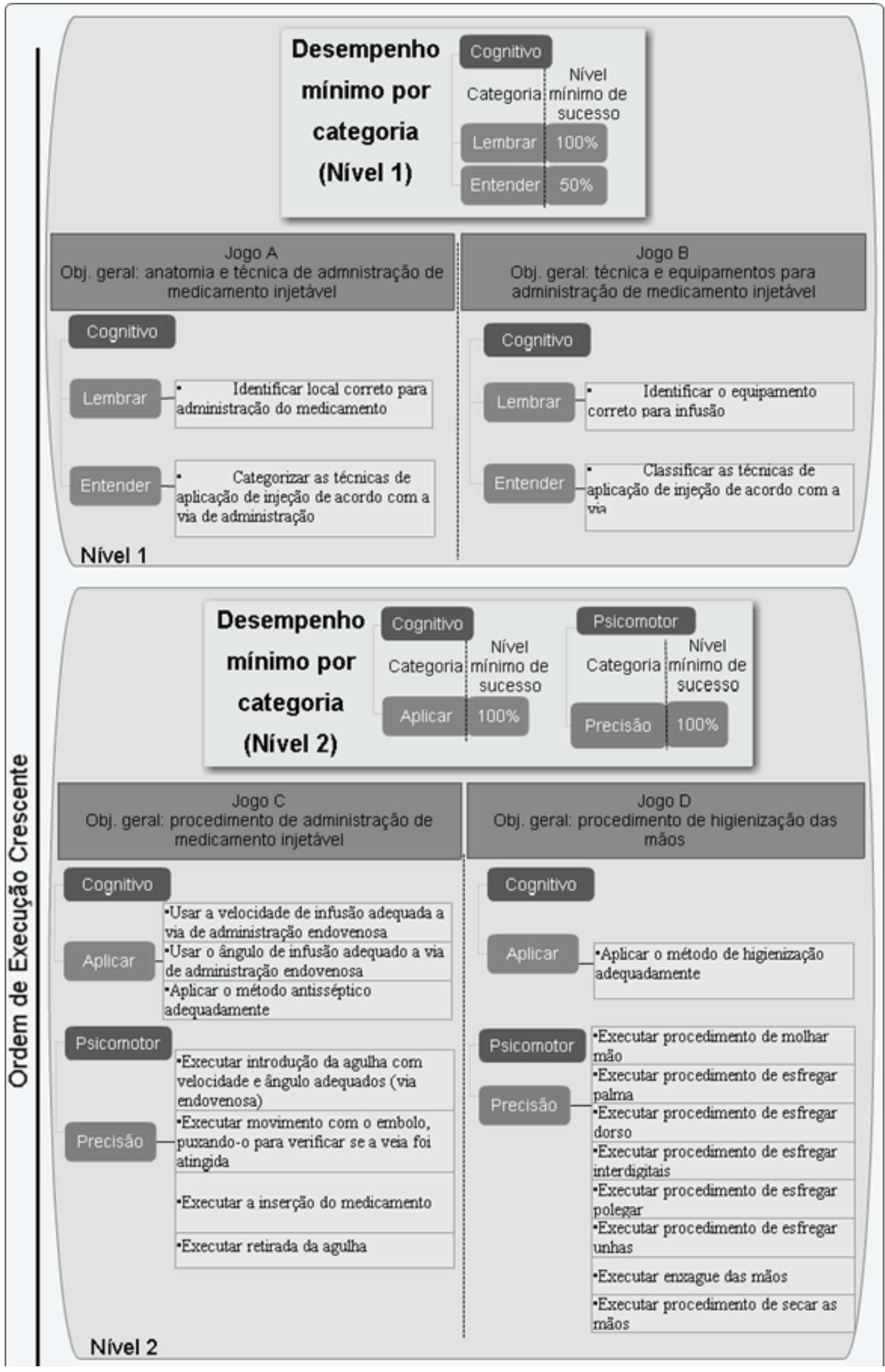




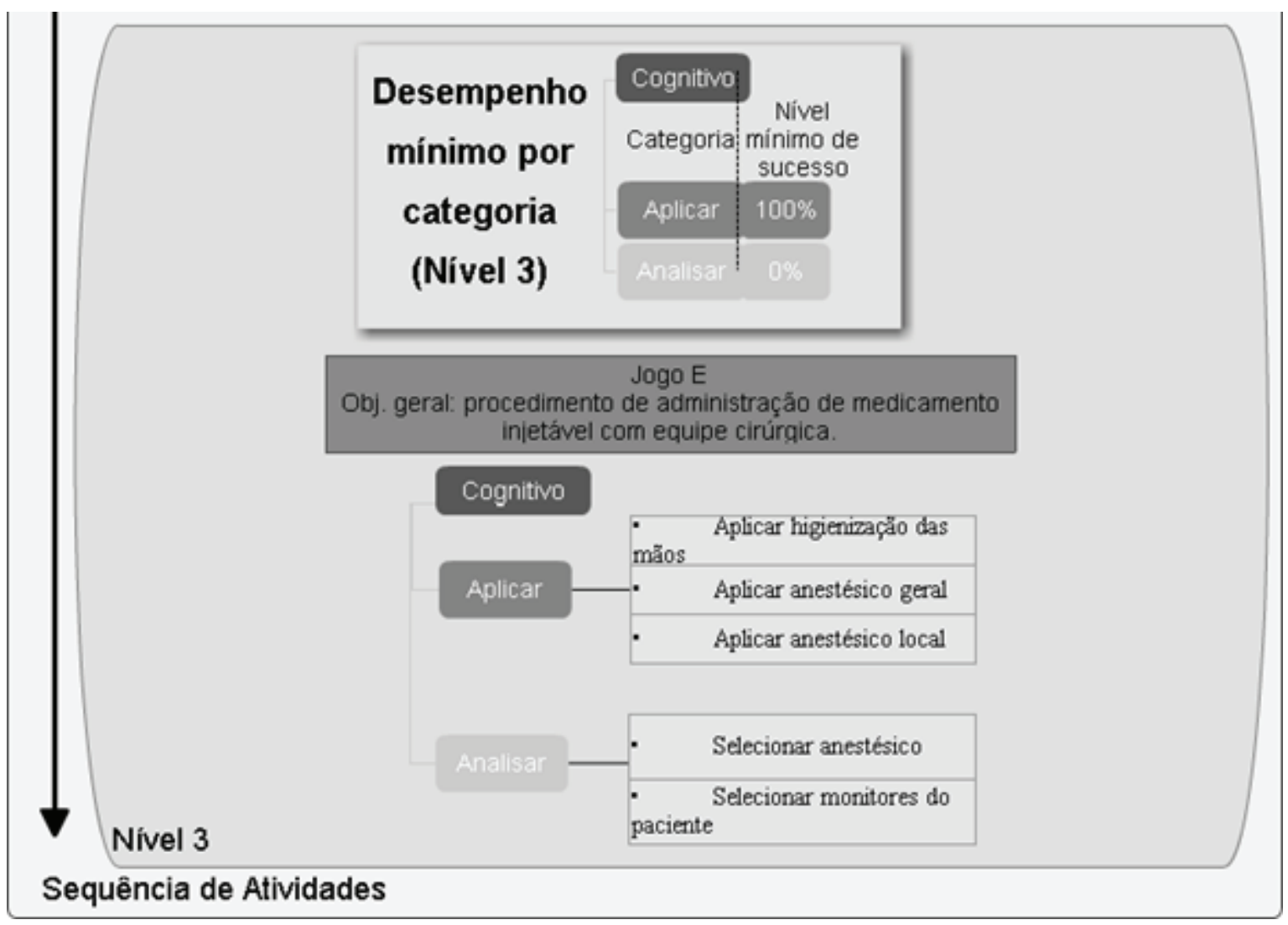

Com essa configuração de avaliação um aluno que tenha sucesso no jogo A e não tenha sucesso no jogo B deverá jogar este último novamente a fim de praticar e demonstrar que conhece ("lembra") o conteúdo. No final, ao completar o último nível, ele conseguirá demonstrar que cumpriu os objetivos da sequência de atividades.

\section{CONSIDERAÇÕES}

Os processos de tomada de decisão por meio da IA estão presentes em diferentes atividades. No contexto dos portais de jogos e simulações, os processos de decisão de máquina também podem auxiliar a avaliação de usuários com possibilidade de controlar mudanças no ambiente.

Os portais de jogos e ambientes virtuais podem ser aproveitados como instrumentos complementares ao processo de formação em saúde, ajudando a organizar atividades e acompanhar o desempenho do usuário durante a realização das atividades planejadas, que podem incluir simulações de habilidades motoras. A partir de alguns instrumentos observados, percebeu-se que os serviços oferecidos pelas plataformas e portais podem incluir o planejamento de atividades e monitoramento. Nesse contexto, o processo de decisão de máquina é usado em processos de avaliação de usuário, auxiliando em análise e controle de atividades.

Nessa linha, este trabalho apresentou um modelo de avaliação que se apoia na Taxonomia dos Objetivos Educacionais para analisar o desempenho dos estudantes considerando aspectos entre 
jogos e ambientes virtuais. Essa taxonomia é utilizada como subsídio que permite a classificação dos objetivos dentro de um portal composto por diferentes jogos e simulações como forma de padronizar a linguagem de comunicação para permitir análises. Assim, o modelo de avaliação fornece uma alternativa para avaliação de desempenho baseada em medidas objetivas de análise de desempenho.

O esquema de avaliação apresentado permite que o mediador personalize o modelo de avaliação para dar mais enfoque os objetivos educacionais do seu planejamento. A partir da definição dos objetivos, o mediador projeta seu esquema de avaliação de desempenho e o modelo, por meio de técnicas de inteligência, realizam as análises para verificação da meta. A possibilidade de customização da avaliação, juntamente com a possibilidade de criação de diferentes sequências de atividades, permite o uso do modelo nas distintas fases da capacitação para auxiliar na formação individual ou em equipe.

O modelo apresentado encontra-se em fase de testes, mas já oferece um meio diferenciado de realizar a avaliação de desempenho do usuário em portais de jogos e AVS a partir da proposta de análise do cumprimento dos objetivos educacionais. Além disso, também permite um processo de avaliação que, embora seja realizado automaticamente, é projetado pelo mediador que o adapta às necessidades dos alunos. Particularmente, essa abordagem é interessante porque permite que sejam consideradas no processo de avaliação as competências do domínio afetivo e psicomotor. Tal característica é relevante em um momento tecnológico no qual se observa a disponibilidade de aplicações na forma de jogos e ambientes virtuais que abordam habilidades que vão além do domínio cognitivo, tais como a destreza motora ${ }^{22}$ e os valores internalizados ${ }^{23}$.

\section{REFERÊNCIAS BIBLIOGRÁFICAS}

1. Taekman JM, Shelley K. Virtual environments in healthcare: immersion, disruption, and flow. International Anesthesiology Clinics. 2010; 48(3):101-21.

2. Machado LS, Moraes RM, Nunes FLS, Costa RME. Serious Games Baseados em Realidade Virtual para Educação Médica. Rev. Brasileira de Educação Médica (Imp.). 2011; $35: 254-262$.

3. Nunes F, Machado LS, Moraes RM. Evolução da Realidade Virtual e Aumentada em Saúde: Uma Reflexão a partir de 15 Anos de SVR. Anais do Symposium on Virtual and Augmented Reality; 2014. p. 220-229.

4. Funge JD. Artificial Intelligence for Computer Games. Wellesley: A.K.Peters. 2004.

5. Nogueira DN, Chaimowicz L, Prates RO. Pingo-An Online Portal for Educational Games with Customizable Content. Anais do XII Simpósio Brasileiro de Jogos e Entretenimento Digital. São Paulo.2013. p.80-89 
6. Levashenko V, Zaitseva E, Kvassay M, Kostolny J.Educational portal with Data Mining support for pupils of primary schools. Digital Technologies (DT). International Conference on. 2013. p.33-38.

7. Čišić D, Čičin-Šain M, Ogrizovič D. mGBL-FLOSS m-learning platform. MIPRO, 2010. Proceedings of the 33rd International Convention, 2010. p.1099-1102.

8. Bamidis PD, Konstantinidis EI, Billis A, Frantzidis C, Tsolaki M, Hlauschek W, et al. A Web services-based exergaming platform for senior citizens: The long lasting memories project approach to e-health care. Engineering in Medicine and Biology Society, Annual International Conf. of the IEEE.2011. p.2505-2509.

9. Wagner G. Simurena-A web portal for open educational simulation. Simulation Conference (WSC), Proceedings of the 2012 Winter. 2012. p.1-12.

10. Barclay AR, Bauman EB, Mihai A, Huggins E, Schaefer ER, Seider S, et al. Games \& Simulation for Healthcare [Internet]. University of Wisconsin-Madison. 2013 [Citado em 2015 Março. Disponível em: http://healthcaregames.wisc.edu/index.php.

11. Bastable SB, Gramet P, Jacobs K, Sopczyk DL. Health professional as educator: Principles of teaching and learning. Sudbury, MA:Jones\& Bartlett. 2011.

12. Anderson LW, Krathwohl DR, Airasian PW, Cruikshank KA, Mayer RE, Pintrich PR, et al. (Eds..) A Taxonomy for Learning, Teaching, and Assessing: A Revision of Bloom's Taxonomy of Educational Objectives. Allyn \& Bacon. Boston, MA (Pearson Education Group). 2001.

13. Ferraz APCM, Belhot RV. Taxonomia de Bloom: revisão teórica e apresentação das adequações do instrumento para definição de objetivos instrucionais. Gest. Prod., São Carlos. 2010. 17(2):421-431.

14. Bloom BS, Engelhart MD, Furst EJ, Hill WH, Krathwohl DR. Taxionomia de Objetivos Educacionais: Domínio Cognitivo. Tradução Sant'Anna, F.M. 8. ed. Porto Alegre: Editora Globo. 1983.

15. Bloom BS, Krathwohl DR, Masia BB. Taxionomia dos Objetivos Educacionais: Domínio Afetivo.Tradução Cunha, J.A. 1. ed. 4ª impressão. Porto Alegre: Editora Globo. 1976.

16. Dave, R. Psychomotor Levels. In R. J. Armstrong (Ed.). Developing and Writing Behavioral Objectives. Tucson, AZ: Educational Innovators Press. 1970.

17. Reilly DE, Oermann M. Behavioral Objectives: Evaluation in Nursing. 3rd. ed. New York, NY: National League for Nursing.1990. 
18. Conklin, J. A taxonomy for learning, teaching and assessing: a revision of Blooms's taxonomy of educational objectives. Educational Horizons, v. 83, n. 3, p. 153-159, 2005.

19. Krathwohl DR. A Revision of Bloom's Taxonomy: An Overview. Theory into Practice. 2002; 41(4):212-218.

20. Ligeza A. Logical Foundations for Rule-based Systems. 2nd edn. Springer, Heidelberg. 2006.

21. Millington I, Funge J. Artificial Intelligence For Games. Morgan Kaufmann. Elsevier. 2009.

22. Machado LS, Zuffo MK. Development and Evaluation of a Simulator of Invasive Procedures in Pediatric Bone Marrow Transplant. Studies in Health Technology and Informatics, Amsterdam. 2003, 94:193-195.

23. Almeida LR, Silva ATMC, Machado LS. Caixa de Pandora: Desenvolvendo afetividade e cognição em um serious game para o enfrentamento da violência contra a mulher. In: Anais do Simpósio Brasileiro de Jogos e Entretenimento Digital - Trilha Cultura, 2013, São Paulo, p. 2330 .

Artigo apresentado em 02-09-15

Artigo aprovado em 06-02-16 Artigo publicado no sistema em 21-02-16 